PROCEEDINGS OF THE

AMERICAN MATHEMATICAL SOCIETY

Volume 131, Number 9, Pages 2641-2647

S 0002-9939(03)06952-1

Article electronically published on February 20, 2003

\title{
CASTELNUOVO-MUMFORD REGULARITY OF SIMPLICIAL SEMIGROUP RINGS WITH ISOLATED SINGULARITY
}

\author{
JÜRGEN HERZOG AND TAKAYUKI HIBI
}

(Communicated by Bernd Ulrich)

\begin{abstract}
Let $S=K\left[x_{1}, \ldots, x_{n}\right]$ be the polynomial ring in $n \geq 2$ variables over a field $K$ and $\mathfrak{m}$ its graded maximal ideal. Let $f_{1}, \ldots, f_{m} \in S$ be homogeneous polynomials of degree $d-1 \geq 2$ generating an $\mathfrak{m}$-primary ideal, and let $g_{1}, \ldots, g_{r} \in S$ be arbitrary homogeneous polynomials of degree $d$. In the present paper it will be proved that the Castelnuovo-Mumford regularity of the standard graded $K$-algebra $A=K\left[\left\{f_{i} x_{j}\right\}_{\substack{i=1, \ldots, m \\ j=1, \ldots, n}}, g_{1}, \ldots, g_{r}\right]$ is at most $(d-2)(n-1)$. By virtue of this result, it follows that the regularity of a simplicial semigroup ring $K[C]$ with isolated singularity is at most $e(K[C])-\operatorname{codim}(K[C])$, where $e(K[C])$ is the multiplicity of $K[C]$ and $\operatorname{codim}(K[C])$ is the codimension of $K[C]$.
\end{abstract}

\section{INTRODUCTION}

Castelnuovo-Mumford regularity of graded rings and ideals is one of the most active research topics in computational commutative algebra and computational algebraic geometry.

Let $S=K\left[x_{1}, \ldots, x_{n}\right]$ denote the polynomial ring in $n \geq 2$ variables over a field $K$, and let $M$ be a finitely generated graded $S$-module. If

$$
\cdots \longrightarrow F_{j} \longrightarrow \cdots \longrightarrow F_{0} \longrightarrow M \longrightarrow 0
$$

is the graded minimal free $S$-resolution of $M$, then the Castelnuovo-Mumford regularity $\operatorname{reg}(M)$ of $M$ is the nonnegative integer $\operatorname{reg}(M)=\max \left\{b_{j}-j: j=0,1, \ldots\right\}$, where $b_{j}$ is the maximal degree of the generators of the graded free $S$-module $F_{j}$.

We are especially interested in the Castelnuovo-Mumford regularity of the standard graded $K$-algebra $A=S / I$, where $I$ is a homogeneous ideal of $S$. Eisenbud and Goto conjectured in their paper [3] that if $A$ is an integral domain, then $\operatorname{reg}(A)$ satisfies the inequality

$$
\operatorname{reg}(A) \leq e(A)-\operatorname{codim}(A),
$$

where $e(A)$ is the multiplicity of $A$ and $\operatorname{codim}(A)$ is the codimension of $A$. The Eisenbud-Goto conjecture turns out to be true in several special cases considered

Received by the editors April 1, 2002.

2000 Mathematics Subject Classification. Primary 13D45; Secondary 52A38.

Key words and phrases. Castelnuovo-Mumford regularity, Eisenbud-Goto conjecture, simplicial semigroup ring, isolated singularity. 
in algebraic geometry; see [5] and [6]. However, the conjecture is widely open in general; even in the case that $A$ is an affine semigroup ring.

Let $\mathfrak{m}$ denote the graded maximal ideal of $S$. In the present paper, we pay attention to the Castelnuovo-Mumford regularity of the standard graded $K$-algebra $A=K\left[f_{1}, \ldots, f_{m}\right]$, where $I=\left(f_{1}, \ldots, f_{m}\right) \subset S$ is a homogeneous ideal generated in degree $d$ such that $I^{k}=\mathfrak{m}^{d k}$ for some $k>0$. For such a $K$-algebra $A$ one has $e(A)=d^{n-1}$ and $\operatorname{codim}(A) \leq\left(\begin{array}{c}d+n-1 \\ n-1\end{array}\right)-n$.

For a particular class of such $K$-algebras we can bound the regularity. This is shown in Theorem 1.1 As a consequence we obtain in Corollary 1.3 that for such a $K$-algebra $A$, one has $\operatorname{reg}(A) \leq e(A)-\operatorname{codim}(A)$, if $n \geq 3$.

Recently, in Hoa and Stückrad [4] the regularity of simplicial semigroup rings was studied. Their work strongly stimulates the research to find reasonable classes of simplicial semigroup rings satisfying inequality (1). As a conclusion of Corollary 1.3 and a simple counting argument, we show in our final Corollary 2.2 that the Eisenbud-Goto conjecture holds for simplicial semigroup rings with isolated singularity.

\section{Regularity of CERTAin GRADED RINGS GENERATED BY $d$-FORMS}

Let $K$ be a field and $S=K\left[x_{1}, \ldots, x_{n}\right]$ the polynomial ring in $n \geq 2$ variables over $K$ with the graded maximal ideal $\mathfrak{m}=\left(x_{1}, \ldots, x_{n}\right)$.

Theorem 1.1. Let $f_{1}, \ldots, f_{m} \in S$ be homogeneous polynomials of degree $d-1 \geq 2$ generating an $\mathfrak{m}$-primary ideal, and let $g_{1}, \ldots, g_{r} \in S$ be arbitrary homogeneous polynomials of degree $d$. Then the regularity of the standard graded $K$-algebra $A=$ $K\left[\left\{f_{i} x_{j}\right\}_{\substack{i=1, \ldots, m \\ j=1, \ldots, n}}, g_{1}, \ldots, g_{r}\right]$ is at most $(d-2)(n-1)$.

Proof. We may assume that $K$ is an infinite field. Let $J=\left(f_{1}, \ldots, f_{m}\right)$. Then there exists an ideal $L \subset J$ generated by a regular sequence of length $n$ consisting of elements of degree $d-1$. For a finite length graded $S$-module $N$ we set $s(N)=$ $\max \left\{i: N_{i} \neq 0\right\}$. It is known that $\operatorname{reg}(N)=s(N)$. Therefore for $k$ it follows that

$$
\operatorname{reg}\left(J^{k}\right)=\operatorname{reg}\left(S / J^{k}\right)+1 \leq \operatorname{reg}\left(S / L^{k}\right)+1=\operatorname{reg}\left(L^{k}\right) .
$$

Let $L=\left(\ell_{1}, \ldots, \ell_{n}\right)$. Since $L$ is generated by a regular sequence of length $n$ of elements of degree $d-1$, the resolution of $L^{k}$ is given by the Eagon-Northcott complex (see, e.g., 2] ) attached to the $(d-1) \times(n+d-2)$ matrix

$$
\left(\begin{array}{cccccc}
\ell_{1} & \cdots & \ell_{n} & 0 & \cdots & 0 \\
0 & \ell_{1} & \cdots & \ell_{n} & \cdots & 0 \\
\vdots & & \ddots & & \ddots & \vdots \\
0 & \cdots & 0 & \ell_{1} & \cdots & \ell_{n}
\end{array}\right) .
$$

It follows immediately from this resolution that $\operatorname{reg}\left(L^{k}\right)=(d-1) k+(d-2)(n-1)$.

For the convenience of the reader we give a direct proof of this fact: The ideal $\mathfrak{m}^{k}=\left(x_{1}, \ldots, x_{n}\right)^{k}$ has a $K$-linear resolution. In particular, the generators in the last step of the resolution are of degree $k+n-1$. Consider the flat map $\varphi: S \rightarrow S$ with $\varphi\left(x_{i}\right)=\ell_{i}$ for $i=1, \ldots, n$. Then $\varphi\left(\mathfrak{m}^{k}\right)=L^{k}$, and so the resolution of $L^{k}$ is obtained from that of $\mathfrak{m}^{k}$ by replacing each $x_{i}$ with $\ell_{i}$. This implies that all the shifts are multiplied by $d-1$. Hence the generators in the last step of the resolution of $L^{k}$ are of degree $(k+n-1)(d-1)$. From this we conclude that $\operatorname{reg}\left(L^{k}\right)=(k+n-1)(d-1)-(n-1)=(d-1) k+(d-2)(n-1)$. 
Let $I=J \mathfrak{m}$. We claim that $I^{k}$ has a linear resolution if $k \geq(d-2)(n-1)$. In fact, $I^{k}=J^{k} \mathfrak{m}^{k}=\left(J^{k}\right)_{\geq(d-1) k+k}$, where for a graded module $M$ we set $M_{\geq j}=\bigoplus_{i \geq j} M_{i}$. Recall from [3] (or [1, Theorem 4.3.1]) that

$$
\operatorname{reg} M=\min \left\{j: M_{\geq j} \text { has a linear resolution }\right\} .
$$

It follows that $I^{k}$ has a linear resolution if and only if $(d-1) k+k \geq \operatorname{reg}\left(J^{k}\right)$. In particular, $I^{k}$ has a linear resolution if $(d-1) k+k \geq(d-1) k+(d-2)(n-1)$, namely if $k \geq(d-2)(n-1)$.

Next we notice that an m-primary ideal $H$ generated in one degree, say $h$, has a linear resolution if and only if it is a power of $\mathfrak{m}$. To see why this is true, we observe that $H$ has a linear resolution if and only if $\operatorname{reg}(H)=h$. But $\operatorname{reg}(H)=$ $s(S / H)+1=h$ if and only if $H=\mathfrak{m}^{h}$.

Applied to our situation we conclude that $I^{k}=\mathfrak{m}^{d k}$ for $k \geq(d-2)(n-1)$. This implies that $A_{k}=S_{d k}$ for all $k \geq(d-2)(n-1)$. Let $A^{*}$ be the integral closure of $A$. Then $A^{*}=S^{(d)}$, the $d$ th Veronese subring of $S$, and $A^{*} / A$ is of finite length with $s\left(A^{*} / A\right) \leq(d-2)(n-1)-1$.

Let $\mathfrak{n}$ be the graded maximal ideal of $A$. Local cohomology applied to the exact sequence

$$
0 \longrightarrow A \longrightarrow A^{*} \longrightarrow A^{*} / A \longrightarrow 0
$$

yields that $H_{\mathfrak{n}}^{0}\left(A^{*} / A\right)=H_{\mathfrak{n}}^{1}(A)$ and $H_{\mathfrak{n}}^{i}(A)=H_{\mathfrak{n}}^{i}\left(A^{*}\right)$ for $i>1$. Since $A^{*}$ is Cohen-Macaulay, one also has $H_{\mathfrak{n}}^{i}\left(A^{*}\right)=0$ for $i<d=\operatorname{dim}(A)=\operatorname{dim}\left(A^{*}\right)$. Hence $\operatorname{reg}(A)=\max \left\{\operatorname{reg}\left(A^{*}\right), \operatorname{reg}\left(A^{*} / A\right)+1\right\}$. Since $\operatorname{reg}\left(A^{*} / A\right)=s$, it follows that

$$
\operatorname{reg}(A)=\max \left\{\operatorname{reg}\left(A^{*}\right), s\left(A^{*} / A\right)+1\right\} \leq(d-2)(n-1),
$$

since the regularity of the Cohen-Macaulay algebra $A^{*}$ is $n$ plus its $a$-invariant, and hence at most $n-1$, and since $n-1 \leq(d-2)(n-1)$ because $d \geq 3$.

Theorem 1.1 suggests the following question: Let $f_{1}, \ldots, f_{m}$ be homogeneous polynomials of degree $d$ and suppose that $A_{k}=S_{d k}$ for some $k$. Does this imply that $\operatorname{reg}(A) \leq(d-2)(n-1)$ ?

We shall need the following numerical result.

Lemma 1.2. If $n \geq 3$ and $d \geq 3$, then $(d-2)(n-1) \leq d^{n-1}-\left(\left(\begin{array}{c}n+d-1 \\ n-1\end{array}\right)-n\right)$.

Proof. Replace $n-1$ with $n$ in the required inequality, and what we must prove is the inequality

$$
(d-2) n \leq d^{n}-\left(\left(\begin{array}{c}
n+d \\
n
\end{array}\right)-(n+1)\right)
$$

for $n \geq 2$ and $d \geq 3$. Inequality (2) is equivalent to the inequality

$$
d^{n}-\left(\begin{array}{c}
n+d \\
n
\end{array}\right) \geq(d-2) n-(n+1)
$$

Thus we must prove the inequality

$$
d^{n}-\prod_{i=1}^{n}\left(1+\frac{d}{i}\right) \geq n d-3 n-1
$$

for $n \geq 2$ and $d \geq 3$.

Fix $d \geq 3$. By using induction on $n \geq 2$ we will prove (3).

If $n=2$, then the inequality (3) coincides with $(d-3)(d-4) \geq 0$. 
Let $n \geq 2$ and suppose that the inequality (3) is true. Inequality (3) for $n+1$ then follows from the computation below:

$$
\begin{aligned}
& d^{n+1}-\prod_{i=1}^{n+1}\left(1+\frac{d}{i}\right) \\
= & \left(d^{n+1}-d^{n}\right)-\left(\prod_{i=1}^{n+1}\left(1+\frac{d}{i}\right)-\prod_{i=1}^{n}\left(1+\frac{d}{i}\right)\right)+d^{n}-\prod_{i=1}^{n}\left(1+\frac{d}{i}\right) \\
\geq & d^{n}(d-1)-\frac{d}{n+1} \prod_{i=1}^{n}\left(1+\frac{d}{i}\right)+(n d-3 n-1) \\
\geq & d^{n}(d-1)-\frac{d^{n}}{n+1}(1+d)+(n d-3 n-1) \\
= & d^{n}\left((d-1)-\frac{d+1}{n+1}\right)+(n d-3 n-1) \\
\geq & d^{n}\left((d-1)-\frac{d+1}{2}\right)+(n d-3 n-1) \\
= & \frac{d^{n}(d-3)}{2}+(n d-3 n-1) \\
\geq & (d-3)+(n d-3 n-1) \\
= & (n+1) d-3(n+1)-1 .
\end{aligned}
$$

Corollary 1.3. Let $A$ be the $K$-algebra as defined in Theorem 1.1, and assume that $n \geq 3$. Then

$$
\operatorname{reg}(A) \leq e(A)-\operatorname{codim}(A)
$$

Proof. If $n \geq 3$, then the assertion follows from Theorem 1.1 together with Lemma 1.2 because $e(A)=e\left(S^{(d)}\right)=d^{n-1}$ and $\operatorname{codim}(A) \leq\left(\begin{array}{c}n+d-1 \\ n-1\end{array}\right)-n$.

\section{Simplicial SEMIGROUP RINGS WITH ISOLATED SINGULARITY}

Let $C$ be a positive affine semigroup of rank $n$, i.e., the associated group $\mathbb{Z} C$ is isomorphic to $\mathbb{Z}^{n}$ and $\{0\}$ is the only subgroup contained in $C$. Let $G$ be the minimal set of generators of $C$. We say that $C$ is standard graded if there exists a hyperplane $H \subset \mathbb{Z} C \otimes_{\mathbb{Z}} \mathbb{Q}$ such that $G \subset H$. Let $P$ be the convex hull of $G$ in $\mathbb{Z} C \otimes_{\mathbb{Z}} \mathbb{Q}$. We say that $C$ is simplicial if $P$ is a simplex. Let $v_{1}, \ldots, v_{n}$ be the vertices of $P$. After the choice of a basis of $\mathbb{Z} C$, the vertices $v_{i}$ can be identified with integral vectors. Let

$$
A=\left(v_{1}^{t}, \ldots, v_{n}^{t}\right)
$$

be the $n \times n$ matrix whose columns are the transpose of the vertices $v_{i}$. We denote by $A^{*}$ the adjoint matrix of $A$. Let $\delta=\operatorname{det}(A)$. Then $\delta \neq 0$ and $A^{*} A=\delta E_{n}$, where $E_{n}$ is the unit matrix of size $n$.

Let $\varphi: \mathbb{Z} C \rightarrow \mathbb{Z}^{n}$ be the linear map associated with $A^{*}$. It then follows that the simplex $P^{\prime}=\varphi(P) \subset \mathbb{Z}^{n}$ has the vertices $\delta \varepsilon_{i}$, where $\varepsilon_{i}$ denotes the $i$ th standard unit vector of $\mathbb{Q}^{n}$. Let $C^{\prime}=\varphi(C)$ and $G^{\prime}=\varphi(G)$. Note that $C^{\prime}$ is isomorphic to $C$ and that $G^{\prime}$ is the minimal set of generators of $C^{\prime}$ with $\left\{\delta \varepsilon_{1}, \ldots, \delta \varepsilon_{n}\right\} \subset G^{\prime} \subset P^{\prime}$. Let $t$ be the greatest common divisor of all the components of all the vectors belonging to $G^{\prime}$, and set $d=\delta / t$. Denote by $C^{\prime \prime} \subset \mathbb{Z}$ the semigroup generated by $G^{\prime \prime}=\frac{1}{t} G^{\prime}$. 
Then it is clear that the convex hull of $G^{\prime \prime}$ is the simplex with vertices $d \varepsilon_{1}, \ldots, d \varepsilon_{n}$, that $C^{\prime \prime} \cong C$ and that $\left[\mathbb{Z}^{n}: \mathbb{Z} C^{\prime \prime}\right]=d$. We call $d$ the index of $C$. It is in fact an invariant of $C$, i.e., does not depend on the particular basis of $\mathbb{Z} C$ which was chosen to define the matrix $A$. We say that the simplicial semigroup $C^{\prime \prime}$ is standard embedded.

Theorem 2.1. Let $C$ be a simplicial semigroup of rank $n>1$ with index $d>2$. Let $K$ be a field and $K[C]$ the semigroup ring associated with $C$. Suppose that $K[C]$ is a $K$-algebra with isolated singularity. Then

$$
\operatorname{reg}(K[C]) \leq(d-2)(n-1) .
$$

Proof. Since $K[C] \cong K\left[C^{\prime}\right]$ we may assume that the embedding of $C$ itself is standard. Let $[n]=\{1, \ldots, n\}$. Write $\mathbf{x}^{\mathbf{w}}=x_{1}^{w_{1}} \cdots x_{n}^{w_{n}}$ if $\mathbf{w}=\left(w_{1}, \ldots, w_{n}\right) \in \mathbb{Z}^{n}$.

For each $1 \leq i \neq j \leq d$, we write $q_{i}^{j}(\geq 1)$ for the biggest integer satisfying

$$
d \varepsilon_{i}+\frac{1}{q_{i}^{j}}\left(d \varepsilon_{j}-d \varepsilon_{i}\right) \in G
$$

Since the localization

$$
K[C]_{x_{i}^{d}}=K\left[x_{i}^{d}, \frac{1}{x_{i}^{d}}\right]\left[\left\{\frac{\mathbf{x}^{\mathbf{w}}}{x_{i}^{d}}\right\}_{\mathbf{w} \in G}\right]=K\left[x_{i}^{d}, \frac{1}{x_{i}^{d}}\right]\left[\left\{\mathbf{x}^{\mathbf{w}-d \varepsilon_{i}}\right\}_{\mathbf{w} \in G}\right]
$$

is regular if and only if

$$
K[C]_{x_{i}^{d}}=K\left[x_{i}^{d}, \frac{1}{x_{i}^{d}}\right]\left[\left\{\mathbf{x}^{\frac{1}{q_{i}^{j}}\left(d \varepsilon_{j}-d \varepsilon_{i}\right)}\right\}_{j \in[n] \backslash\{i\}}\right],
$$

and since $K[C]$ is a $K$-algebra with isolated singularity, it follows that, for any $\mathbf{w} \in G$ and for any $1 \leq i \leq n$, there exists $0 \leq p_{j} \in \mathbb{Z}, j \in[n] \backslash\{i\}$, such that

$$
\mathbf{w}-d \varepsilon_{i}=\sum_{j \in[n] \backslash\{i\}} \frac{p_{j}}{q_{i}^{j}}\left(d \varepsilon_{j}-d \varepsilon_{i}\right) .
$$

This simple observation yields the crucial result that $q_{i}^{j}=q_{k}^{\ell}$ for all $i, j, k, \ell$ with $i \neq j$ and $k \neq \ell$. In fact, in case of $1 \leq i \neq j \leq n$, since

$$
\left(d \varepsilon_{j}+\frac{1}{q_{j}^{i}}\left(d \varepsilon_{i}-d \varepsilon_{j}\right)\right)-d \varepsilon_{i}=\frac{p}{q_{i}^{j}}\left(d \varepsilon_{j}-d \varepsilon_{i}\right), \quad 0 \leq p \in \mathbb{Z},
$$

one has $q_{i}^{j}=q_{j}^{i}\left(q_{i}^{j}-p\right)$. Thus $q_{j}^{i}$ divides $q_{i}^{j}$. Similarly, $q_{i}^{j}$ divides $q_{j}^{i}$. Hence $q_{i}^{j}=q_{j}^{i}$. Also, in case of $i, k, \ell \in[n]$ with $i \neq k, k \neq \ell$ and $i \neq \ell$, since

$$
d \varepsilon_{k}-d \varepsilon_{i}+\frac{1}{q_{k}^{\ell}}\left(d \varepsilon_{\ell}-d \varepsilon_{k}\right)=\sum_{j \in[n] \backslash\{i\}} \frac{p_{j}}{q_{i}^{j}}\left(d \varepsilon_{j}-d \varepsilon_{i}\right), \quad 0 \leq p_{j} \in \mathbb{Z},
$$

one has $\frac{1}{q_{k}^{\ell}}=\frac{p_{\ell}}{q_{i}^{\ell}}$. Thus $q_{k}^{\ell}$ divides $q_{i}^{\ell}$. Similarly, $q_{i}^{\ell}$ divides $q_{k}^{\ell}$. Hence $q_{i}^{\ell}=q_{k}^{\ell}$.

Let $q=q_{i}^{j}$ for all $1 \leq i \neq j \leq n$. Then $0<\frac{d}{q} \in \mathbb{Z}$ divides each component of any vector belonging to $G$. Since the embedding of $C$ is standard, it follows that $q=d$.

We now conclude that

$$
K[C]=K\left[\left\{x_{i}^{d-1} x_{j}\right\}_{\substack{i=1, \ldots, n \\ j=1, \ldots, n}}, g_{1}, \ldots, g_{r}\right],
$$

where $g_{1}, \ldots, g_{r}$ are monomials of degree $d$. Hence we are in the situation of Theorem [1.1 with $f_{i}=x_{i}^{d-1}$ for $i=1, \ldots, n$. 
The following final result follows partly from Corollary 1.3

Corollary 2.2. Let $K[C]$ be a simplicial semigroup ring with isolated singularity. Then

$$
\operatorname{reg}(K[C]) \leq e(K[C])-\operatorname{codim}(K[C]) .
$$

Proof. Fix a standard embedding of $C$. Let $\operatorname{rank} C=n$. For $n \geq 3$, the assertion follows from (4) and Corollary 1.3 .

Now let $n=2$. Then

$$
K[C]=K\left[\left\{x_{1}^{d-a_{i}} x_{2}^{a_{i}}\right\}_{i=0, \ldots, r+1}\right],
$$

with $0=a_{0}<1=a_{1}<a_{2}<\cdots<d-1=a_{r}<d=a_{r+1}$.

Therefore, $e(K[C])=d$, and $\operatorname{codim}(K[C])=r$. Thus we need to show that $\operatorname{reg}(K[C]) \leq d-r$, or equivalently, that

$$
K[C]_{d-r}=K\left[\left\{x_{1}^{(d-r) d-j} x_{2}^{j}\right\}_{j=0, \ldots,(d-r) d}\right] .
$$

Set $k=d-r$, and let $X=\left\{j: x_{1}^{k d-j} x_{2}^{j} \in K[C]_{d-r}\right\}$. Since $a_{0}=0$, it follows that

$$
X=\left\{\sum_{i=1}^{r+1} k_{i} a_{i}: k_{i} \geq 0, \quad \sum_{i=1}^{r+1} k_{i} \leq k\right\},
$$

and we have to show that $X=\{0, \ldots, k d\}$.

For any two integers $a \leq b$ we set $[a, b]=\{c \in \mathbb{Z}: a \leq c \leq b\}$. Fix a number $j \in\{0, \ldots, k\}$. Then $a_{i}+j d \in X \cap[j d,(j+1) d]$ for $i=0, \ldots, r+1$.

Next we notice that $a_{i}+j a_{r+1}+l a_{1}=a_{i}+j d+l \in X$ for $l=0, \ldots, k-1-j$, and that $a_{i+1}+l a_{r}+(j-l) a_{r+1}=a_{i+1}+j d-l \in X$ for $l=0, \ldots, j$. Thus we see that

$$
\left[a_{i}+j d, a_{i}+j d+(k-1-j)\right] \cup\left[a_{i+1}+j d-j, a_{i+1}+j d\right] \subset X .
$$

Since

$\left(a_{i+1}+j d-j\right)-\left(a_{i}+j d+(k-1-j)\right)=\left(a_{i+1}-a_{i}\right)-(k-1) \leq(d-r)-(d-r-1)=1$,

it follows that

$$
\left[a_{i}+j d, a_{i+1}+j d\right]=\left[a_{i}+j d, a_{i}+j d+(k-1-j)\right] \cup\left[a_{i+1}+j d-j, a_{i+1}+j d\right],
$$

so that by (5), $\left[a_{i}+j d, a_{i+1}+j d\right] \in X$ for all $i=0, \ldots, r$ and all $j=0, \ldots, k$. Since $[0, k d]=\bigcup_{\substack{i=0, \ldots, r \\ j=0, \ldots, k}}\left[a_{i}+j d, a_{i+1}+j d\right]$, the assertion follows.

\section{REFERENCES}

[1] W. Bruns and J. Herzog, "Cohen-Macaulay rings," Revised Edition, Cambridge University Press, 1996.

[2] W. Bruns and U. Vetter, "Determinantal rings," Lecture Note in Mathematics 1237, SpringerVerlag, 1988. MR 89i:13001

[3] D. Eisenbud and S. Goto, Linear free resolutions and minimal multiplicity, J. Algebra 88 (1984), 89 - 133. MR 85f:13023

[4] L.T. Hoa and J. Stückrad, Castelnuovo-Mumford regularity of simplicial toric rings, preprint, 2001. 
[5] L. Gruson, R. Lazarsfeld and C. Peskine, On a theorem of Castelnuovo, and the equations defining space curves, Inv. Math. 72 (1983), 491 - 506. MR 85g:14033

[6] R. Lazarsfeld, A sharp Castelnuovo bound for smooth surfaces, Duke Math. J. 55 (1987), 423 - 429. MR 89d:14007

FB6 Mathematik und Informatik, Universität - GHS - Essen, Postfach 103764, 45117 Essen, GeRmany

E-mail address: mat300@uni-essen.de

Department of Pure and Applied Mathematics, Graduate School of Information SciEnce and Technology, Osaka University, Toyonaka, Osaka 560-0043, Japan

E-mail address: hibi@math.sci.osaka-u.ac.jp 